\title{
Sleep Quality and Quality of Life in Adults With Type 2 Diabetes
}

\author{
Dr Faith S. Luyster, PhD and Dr Jacqueline Dunbar-Jacob, PhD, RN \\ University of Pittsburgh, School of Nursing, Pittsburgh, PA
}

\begin{abstract}
Purpose-The purpose of this study was to examine the relationship between sleep quality and health-related and diabetes-related quality of life in adults with type 2 diabetes.

Methods-Three hundred individuals with type 2 diabetes (mean age 63.9 years) completed questionnaires assessing sleep quality, health-related quality of life, diabetes-related quality of life, comorbidities, and depressive symptoms.

Results-More than half of the participants (55\%) were "poor sleepers" according to the Pittsburgh Sleep Quality Index. After controlling for covariates, poor sleep quality was found to be a significant predictor of lower health-related quality of life as indicated by lower scores on both the physical and mental component scores of the Medical Outcomes Study 36-item Short Form health survey (SF-36). Poor sleep quality was also associated with worse diabetes-related quality of life, in particular lower scores on the Diabetes Quality of Life measure total score and the Satisfaction With Treatment and Diabetes Impact subscale scores.
\end{abstract}

Conclusions-These results suggest that poor sleep is common in type 2 diabetes and may adversely impact quality of life. Interventions to improve sleep hygiene can be suggested to patients by diabetes educators as part of diabetes self-management education programs.

Type 2 diabetes is one of the most common chronic diseases in the United States, affecting 23.6 million people. ${ }^{1}$ In adults aged 60 years of age or older, the prevalence of type 2 diabetes is more than $20 \%$. Diabetes-related complications, including cardiovascular disease, kidney disease, neuropathy, loss of vision, and lower extremity amputation, contribute significantly to the increased morbidity and mortality among persons with type 2 diabetes and cause a substantial economic strain on the health care system. ${ }^{2}$

Type 2 diabetes inflicts a significant burden in terms of disability and impaired quality of life. ${ }^{3,4}$ Persons with type 2 diabetes report lower health-related quality of life (HRQOL) than the general population ${ }^{5}$. HRQOL is an important outcome for persons with type 2 diabetes, as it has been used to evaluate the impact of the disease and its treatment on individuals and health care costs. Worse HRQOL is associated with higher overall mortality in persons with type 2 diabetes. ${ }^{6}$ The disease itself can have a negative impact on quality of life. ${ }^{7}$ Several factors have been identified as predictors of HRQOL and diabetes-related quality of life in type 2 diabetes, including older age, female sex, depressive symptoms, number of diabetic complications, presence of comorbidities, and insulin use. ${ }^{7-11}$

\footnotetext{
(C) 2011 The Author(s)

Correspondence to Faith S. Luyster, University of Pittsburgh, 3500 Victoria Building, Room 360C, Pittsburgh, PA 15261 (luysterfs@upmc.edu).

The authors have no conflicts of interest to disclose.

For reprints and permission queries, please visit SAGE's Web site at http://www.sagepub.com/journalsPermissions.nav.
} 
Poor sleep is a common feature of type 2 diabetes. ${ }^{12}$ Difficulty initiating and maintaining sleep, daytime sleepiness, and poor sleep quality have been reported by persons with type 2 diabetes. ${ }^{13-15}$ These sleep disturbances are more frequent in those with type 2 diabetes than in age- and sex-matched controls. ${ }^{12,16}$ Although sleep disturbances have been shown to decrease HRQOL in several chronic illnesses, ${ }^{17-20}$ the effect of poor sleep on HRQOL and disease-related quality in type 2 diabetes has not been previously examined. The objective of this study was to assess the impact of sleep quality on HRQOL and disease-related quality of life in persons with type 2 diabetes.

\section{Methods}

\section{Study Population}

The data presented here were collected as part of a randomized clinical intervention trial that examined the impact of a counseling intervention on medication adherence among adults with type 2 diabetes and concurrent hypertension and hyperlipidemia (PI: J. Dunbar-Jacob). For the parent study, 345 patients over the age of 40 who had physician-diagnosed type 2 diabetes for at least 1 year and were taking oral medication for at least 2 of the 3 diagnoses were enrolled in the study. This article presents a secondary analysis of descriptive crosssectional data collected at the baseline assessment in the intervention trial. Of the 345 participants enrolled in the original study, 45 were excluded from analysis because of missing data on the study variables of interest. All participants gave written informed consent, and the study was approved by the institutional review board of the University of Pittsburgh.

\section{Measures}

Sleep quality-Sleep quality was measured using the Pittsburgh Sleep Quality Index (PSQI). ${ }^{21}$ The PSQI assesses quality of sleep during the past month and contains 7 component scales: sleep quality, sleep latency, sleep duration, sleep efficiency, sleep disturbances, use of sleep medication, and daytime dysfunction. Each component is scored from 0 to 3, yielding a global PSQI score ranging from 0 to 21, with higher scores indicating worse sleep quality. A global PSQI score greater than 5 has been found to have a sensitivity of $89.6 \%$ and specificity of $86.5 \%$ in differentiating good from poor sleepers. ${ }^{21}$

Quality of life-The Medical Outcomes Study 36-item Short Form health survey (SF-36) is one of the most widely used questionnaires for assessing health-related quality of life. ${ }^{22}$ The SF-36 contains 36 items evaluating 8 dimensions of health: physical functioning, role limitations due to physical problems, bodily pain, general health perceptions, vitality, social functioning, role limitations due to emotional problems, and mental health. Each dimension is scored on a 0 to 100 scale, with higher scores indicating better functioning. In addition, the SF-36 includes 2 composite summary scores: the physical component score (PCS) and the mental component score (MCS). The PCS and MCS are standardized to a mean (SD) of $50,{ }^{23}$ with lower scores indicating more significant dysfunction. The SF-36 demonstrates high reliability and validity in a variety of diseases. ${ }^{24-26}$

Diabetes-related quality of life was measured using the Diabetes Quality of Life measure (DQOL) ${ }^{27}$ The questionnaire contains 52 items and is composed of 4 subscales: Diabetes Life Satisfaction, Diabetes Impact, Diabetes-Related Worry, and Social/Vocational Worry. The Diabetes Life Satisfaction items assess satisfaction with treatment. The Diabetes Impact items assess impact of treatment. The Diabetes-Related Worry items assess how often participants worry about the future effects of diabetes. The Social/Vocational Worry items assess social and vocational issues, such as getting married, getting a job, and completing their education. The questionnaire was originally developed for use among young adults 
with type 1 diabetes ${ }^{27}$ but has been used in type 2 diabetes populations. ${ }^{28}$ The Social/ Vocational Worry scale will not be included in the analyses because the items are not relevant to mid- to late-life adults. Each item is rated on a 5-point scale, and a total score is obtained by averaging scores for all 4 subscales, with lower scores indicating better QOL. Cronbach's alphas have been reported as 0.46 to 0.92 for the subscales. ${ }^{28}$

Other variables-Age, sex, years since diabetes diagnosis, number of physiciandiagnosed comorbidities, and insulin use were obtained from sociodemographic and comorbiditiy questionnaires developed as part of the Center for Research in Chronic Disorders, University of Pittsburgh's School of Nursing. The number of diabetic complications was determined by assessing whether participants reported having been diagnosed with coronary artery disease, peripheral vascular disease, stroke, nephropathy, retinopathy, or neuropathy. Depressive symptoms were assessed by the Beck Depression Inventory-II (BDI-II). ${ }^{29}$ Scores for the 21 items range from 0 to 3, reflecting the frequency with which the problem occurs. A total score is obtained by summing the scores for the 21 questions (range, $0-63$ ). The BDI-II has high internal consistency (ranging from 0.86 to 0.93 ) and high test-retest reliability. ${ }^{29}$

\section{Data Analysis}

Descriptive statistics for continuous variables were expressed as means and standard deviations (SD). Categorical variables were described as frequencies with percentages for the total sample and by sleep quality status. Spearman correlation coefficients were used to examine associations between continuous variables. Student's $t$ test was used to compare the means of normally distributed variables between "good sleepers" (PSQI $\leq 5)$ and "poor sleepers" (PSQI >5), and the Mann-Whitney $U$ test was used for variables that were not normally distributed. The $\chi^{2}$ test was used to analyze differences between categorical variables. Bonferroni correction for multiple comparisons was used. Twenty-one independent tests were carried out; thus, a $P$ value of $<.002$ was regarded as significant. Multiple linear regression analysis was used to examine the relationships between sleep quality and HRQOL and diabetes-related quality of life (a total of 6 models) while controlling for covariates. The SF-36 MCS and PCS were used as the outcomes variables in preference to the individual domains of the SF-36 to limit the number of regressions. The DQOL total score and the satisfaction, impact, and diabetes-related worry subscales of the DQOL were also used as outcome variables in the regression analyses. Bonferroni corrections were used, where a significance cutoff ( 0.05 divided by 6 models) was set at $<0.008$.

\section{Results}

Sample

A total of 300 individuals with type 2 diabetes were included in the analyses. Characteristics of the participants are presented in Table 1. Mean age of the sample was 63.9 years (SD 10.4 years). More than half the participants were females $(57 \%, \mathrm{n}=171)$. Participants had type 2 diabetes for an average of 9.4 years (SD 7.3 years) and reported an average of 6 (SD 3.1) comorbidities. Fifty-five participants (18\%) had 2 or more diabetic complications. Only 35 participants (12\%) were using insulin.

\section{Sleep Quality}

The mean (SD) PSQI global score was 6.6 (3.7). One hundred and sixty-four participants $(55 \%)$ were poor sleepers (global PSQI $>5$ ). Poor sleepers had significantly poorer SF-36 PCS and MCS and poorer functioning on each of the 8 domains of the SF-36 (all $P<.001$ ) (Table 1). Additionally, poor sleepers had poorer diabetes-related quality of life. There were 
no differences in insulin use between good and poor sleepers (all $P<.001$ ). Using the Bonferroni method to adjust for multiple comparisons, the difference in sleep quality between males and females was not significant $(P=.03)$. Poorer sleep quality was associated with a higher number of comorbidities, greater number of diabetic complications, and higher levels of depressive symptoms but was not associated with age or years since diabetes diagnosis (Table 2). The PSQI global score was significantly correlated with the SF-36 PCS and MCS and the 8 domains of the SF-36 (all $P<.001$ ). Poorer sleep quality was also associated with lower DQOL total score and lower scores on the satisfaction, impact, and diabetes-related worry subscales of the DQOL (all $P<.001$ ).

\section{Quality of Life}

The mean (SD) scores for the SF-36 PCS, MCS, and the 8 domains are shown in Table 1. The mean (SD) SF-36 MCS was 51.1 (11.2), compared with the mean (SD) SF-36 PCS of 42.9 (10.3), suggesting lower mental than physical health quality of life. Accordingly, among the SF-36 domains, participants reported more impairment in the mental health parameters than the physical parameters. In particular, social functioning and role limitations due to emotional problems were the most impaired, with means of 81.7 (22.7) and 81.6 (24.4), respectively. There were significant inverse correlations between age, years since diabetes diagnosis, number of comorbidities, number of diabetic complications, BDI-II, and SF-36 PCS. The SF-36 MCS was positively correlated with age and negatively correlated with number of comorbidities and BDI-II (Table 3). There were no significant differences in SF-36 PCS or MCS between males and females. Participants using insulin had poorer SF-36 PCS than those not using insulin.

The mean DQOL total score was 2.0 (SD 0.6). The mean DQOL satisfaction, impact, and diabetes-related worries subscale scores were 2.4 (SD 0.7), 2.0 (SD 0.6), and 2.0 (0.8), respectively (Table 1). The DQOL total score and the satisfaction, impact, and diabetesrelated worry subscale scores were correlated with age, number of diabetic complications, and BDI-II (all $P<.05$ ). DQOL total score was not significantly associated with number of comorbidities. There were no differences in DQOL total scores or subscale scores between females and males. Participants using insulin had poorer DQOL total score and satisfaction and impact subscale scores than those not using insulin.

\section{Sleep Quality and Quality of Life}

Multivariate analyses controlling for covariates were conducted to examine the associations between sleep quality and HRQOL and diabetes-related quality of life (Tables 4 and 5). After adjustment for age, number of comorbidities, diabetes duration, number of diabetic complications, insulin use, and depressive symptoms, sleep quality was a significant predictor of SF-36 PCS $(\beta=-0.40, P<.05)$. Number of comorbidities $(\beta=-0.88, P<.001)$ and depressive symptoms $(\beta=-0.39, P<.001)$ were significantly associated with SF-36 PCS. Similarly, sleep quality was a significant predictor of SF-36 MCS after controlling for the above covariates $(\beta=-0.46, P<.01)$. Only depressive symptoms were associated with SF-36 MCS $(\beta=-0.91, P<.01)$.

Sleep quality was a significant predictor of DQOL total score $(\beta=0.03, P<.01)$, DQOL satisfaction score $(\beta=0.05, P<.001)$, and DQOL impact score $(\beta=0.04, P<.001)$, after controlling for age, number of comorbidities, diabetes duration, number of diabetic complications, insulin use, and depressive symptoms. Sleep quality was not associated with DQOL diabetes-related worry score even after adjustment for covariates. Depressive symptoms were associated with DQOL total score $(\beta=0.04, P<.001)$, DQOL satisfaction score $(\beta=0.05, P<.001)$, and DQOL impact score $(\beta=0.03, P<.001)$, and age was significantly associated with DQOL satisfaction score $(\beta=-0.02, P<.001)$. 


\section{Discussion}

To our knowledge, this is the first systematic investigation of the impact of poor sleep on quality of life in type 2 diabetes. Using a validated measure of self-reported sleep quality, we found that more than half (55\%) of our participants with type 2 diabetes had poor sleep quality. Comparatively, previous studies have identified between $45 \%$ and $67 \%$ of persons with type 2 diabetes to have poor sleep quality and/or difficulty initiating or maintaining sleep. ${ }^{12-15}$ Poor sleepers in our sample tended to be younger, to be female, to have more comorbidities, and to have higher levels of depressive symptoms than good sleepers. Compared with good sleepers, poor sleepers had significantly worse HRQOL, including all 8 domains of the SF-36 and the SF-36 MCS and PCS, and worse diabetes-related quality of life, including the DQOL total score and the satisfaction, impact, and diabetes-related worry subscales. These findings suggest that poor sleep quality may adversely affect both emotional well-being and physical functioning, contributing to decreased HRQOL and diabetes-related quality of life in persons with type 2 diabetes.

Poor sleep quality and sleep disturbances reported by participants with type 2 diabetes in our study could have been influenced by a number of factors. Pain due to peripheral neuropathy and nocturia are diabetes-related factors that have been associated with frequent nighttime awakenings. ${ }^{14,30}$ On the PSQI, more than half $(61 \%)$ of our sample reported having to get up to use the bathroom during the night 3 or more nights a week and roughly a quarter (28\%) of participants reported having pain that disturbed sleep at least 1 time per week. Other sleep disturbances such as sleep apnea and restless legs syndrome can cause frequent nighttime awakenings. Sleep apnea is common in individuals with type 2 diabetes ${ }^{31}$; thus, it is possible that sleep apnea contributed to poor sleep quality in our study. Although sleep apnea was not assessed in this study, disturbances in sleep due to snoring or coughing 3 or more times a week were reported on the PSQI by $20 \%(\mathrm{n}=59)$ of our sample. Restless legs syndrome has been identified in $18 \%$ to $27 \%$ of adults with type 2 diabetes. ${ }^{12,15,32}$ Those with type 2 diabetes and restless legs syndrome have poorer sleep quality than those without restless legs syndrome. ${ }^{15,33}$ However, one study found no differences in insomnia between adults with type 2 diabetes with and without restless legs syndrome. ${ }^{12}$

In the present study, HRQOL was associated with age, years since diabetes diagnosis, number of comorbidities, number of diabetic complications, insulin use, and depressive symptoms, consistent with previous findings. ${ }^{7-11}$ Sleep quality was associated with number of comorbidities, number of diabetic complications, and depressive symptoms. Poor sleep quality was associated with poorer HRQOL and diabetes-related quality of life after controlling for age, years since diabetes diagnosis, number of comorbidities, number of diabetic complications, insulin use, and depressive symptoms. Sleep quality was not associated with DQOL diabetes-related worry subscale score even after adjustment for covariates. Previous findings in both the general population and chronic disease populations support the link between poor sleep quality and impaired HRQOL. ${ }^{17-20,34}$ Poor sleep quality may negatively affect quality of life related to the satisfaction of treatment and impact of treatment for type 2 diabetes. Further research is needed to clarify the relationship between sleep quality and treatment satisfaction and beliefs, but clearly poor sleep has the potential to reduce patients' satisfaction with treatment and contribute to beliefs that treatment does not have a significant impact on diabetes self-management.

One limitation of this study was that sleep was subjectively assessed and no objective measures of sleep were obtained. Consequently, we are unable to ascertain the underlying causes of insomnia and sleep disturbances in this population. Another limitation is the crosssectional design, which precludes us from establishing cause and effect in the associations examined. Additionally, although the study aimed to control for known potential 
confounding variables of the relationship between sleep quality and quality of life, it was not possible to assess and control for all variables that can affect sleep quality and quality of life.

\section{Conclusions and Implications}

Our results suggest that poor sleep is common among persons with type 2 diabetes and that poor sleep quality is associated with HRQOL and diabetes-related quality of life, in particular satisfaction with and impact of treatment. These findings emphasize the importance of screening new patients for sleep problems, making a referral to a sleep medicine specialist if appropriate, and suggesting sleep hygiene strategies as part of diabetes management. The diabetes educator can play a key role in assessing sleep and providing easy to implement interventions to improve sleep hygiene. ${ }^{35}$

\section{Acknowledgments}

This work was supported by the National Institutes of Health grants DK059048 and NR00392.

\section{References}

1. American Diabetes Association. Total Prevalence of Diabetes and Pre-Diabetes. Alexandria, Va: American Diabetes Association; 2009.

2. Deshpande AD, Harris-Hayes M, Schootman M. Epidemiology of diabetes and diabetes-related complications. Phys Ther. 2008; 88:1254-1264. [PubMed: 18801858]

3. Volpato S, Maraldi C, Fellin R. Type 2 diabetes and risk for functional decline and disability in older persons. Curr Diabetes Rev. 2010; 6:134-143. [PubMed: 20380626]

4. Rubin RR, Peyrot M. Quality of life and diabetes. Diabetes Metab Res Rev. 1999; 15:205-218. [PubMed: 10441043]

5. Ribu L, Hanestad BR, Moum T, Birkeland K, Rustoen T. A comparison of the health-related quality of life in patients with diabetic foot ulcers, with a diabetes group and a nondiabetes group from the general population. Qual Life Res. 2007; 16:179-189. [PubMed: 17091370]

6. Kleefstra N, Landman GW, Houweling ST, et al. Prediction of mortality in type 2 diabetes from health-related quality of life (ZODIAC-4). Diabetes Care. 2008; 31:932-933. [PubMed: 18319325]

7. Sundaram M, Kavookjian J, Patrick JH, Miller LA, Madhavan SS, Scott VG. Quality of life, health status and clinical outcomes in Type 2 diabetes patients. Qual Life Res. 2007; 16:165-177. [PubMed: 17033903]

8. Brown GC, Brown MM, Sharma S, Brown H, Gozum M, Denton P. Quality of life associated with diabetes mellitus in an adult population. J Diabetes Complications. 2000; 14:18-24. [PubMed: 10925062]

9. Glasgow RE, Ruggiero L, Eakin EG, Dryfoos J, Chobanian L. Quality of life and associated characteristics in a large national sample of adults with diabetes. Diabetes Care. 1997; 20:562-567. [PubMed: 9096981]

10. Redekop WK, Koopmanschap MA, Stolk RP, Rutten GE, Wolffenbuttel BH, Niessen LW. Healthrelated quality of life and treatment satisfaction in Dutch patients with type 2 diabetes. Diabetes Care. 2002; 25:458-463. [PubMed: 11874930]

11. Wexler DJ, Grant RW, Wittenberg E, et al. Correlates of health-related quality of life in type 2 diabetes. Diabetologia. 2006; 49:1489-1497. [PubMed: 16752167]

12. Skomro RP, Ludwig S, Salamon E, Kryger MH. Sleep complaints and restless legs syndrome in adult type 2 diabetics. Sleep Med. 2001; 2:417-422. [PubMed: 14592391]

13. Knutson KL, Ryden AM, Mander BA, Van Cauter E. Role of sleep duration and quality in the risk and severity of type 2 diabetes mellitus. Arch Intern Med. 2006; 166:1768-1774. [PubMed: 16983057]

14. Lamond N, Tiggemann M, Dawson D. Factors predicting sleep disruption in Type II diabetes. Sleep. 2000; 23:415-416. [PubMed: 10811386] 
15. Lopes LA, Lins Cde M, Adeodato VG, et al. Restless legs syndrome and quality of sleep in type 2 diabetes. Diabetes Care. 2005; 28:2633-2636. [PubMed: 16249531]

16. Sridhar GR, Madhu K. Prevalence of sleep disturbances in diabetes mellitus. Diabetes Res Clin Pract. 1994; 23:183-186. [PubMed: 7924879]

17. Elder SJ, Pisoni RL, Akizawa T, et al. Sleep quality predicts quality of life and mortality risk in haemodialysis patients: results from the Dialysis Outcomes and Practice Patterns Study (DOPPS). Nephrol Dial Transplant. 2008; 23:998-1004. [PubMed: 17911092]

18. Krishnan V, McCormack MC, Mathai SC, et al. Sleep quality and health-related quality of life in idiopathic pulmonary fibrosis. Chest. 2008; 134:693-698. [PubMed: 18625669]

19. Menefee LA, Frank ED, Doghramji K, et al. Self-reported sleep quality and quality of life for individuals with chronic pain conditions. Clin J Pain. 2000; 16:290-297. [PubMed: 11153783]

20. Nunes DM, Mota RM, de Pontes Neto OL, Pereira ED, de Bruin VM, de Bruin PF. Impaired sleep reduces quality of life in chronic obstructive pulmonary disease. Lung. 2009; 187:159-163. [PubMed: 19399553]

21. Buysse DJ, Reynolds CF III, Monk TH, Berman SR, Kupfer DJ. The Pittsburgh Sleep Quality Index: a new instrument for psychiatric practice and research. Psychiatry Res. 1989; 28:193-213. [PubMed: 2748771]

22. Ware JE Jr, Sherbourne CD. The MOS 36-item short-form health survey (SF-36), I: conceptual framework and item selection. Med Care. 1992; 30:473-483. [PubMed: 1593914]

23. Ware, JE., Jr; Kosinski, M.; Keller, SD. SF-36 Physical and Mental Health Summary Scales: A User's Manual. Boston, Mass: Health Assessment Lab; 1994.

24. Failde I, Ramos I. Validity and reliability of the SF-36 Health Survey Questionnaire in patients with coronary artery disease. J Clin Epidemiol. 2000; 53:359-365. [PubMed: 10785566]

25. Ruta DA, Hurst NP, Kind P, Hunter M, Stubbings A. Measuring health status in British patients with rheumatoid arthritis: reliability, validity and responsiveness of the short form 36-item health survey (SF-36). Br J Rheumatol. 1998; 37:425-436. [PubMed: 9619895]

26. Woodcock AJ, Julious SA, Kinmonth AL, Campbell MJ. Problems with the performance of the SF-36 among people with type 2 diabetes in general practice. Qual Life Res. 2001; 10:661-670. [PubMed: 11871587]

27. Jacobson, AM.; deGroot, M.; Samson, J. Quality of life in behavorial medicine research. In: Dimsdale, JE.; Baum, A., editors. Perspectives on Behavioral Medicine Series. Hillsdale, NJ: Lawrence Erlbaum; 1995. p. 241-262.

28. Jacobson AM, de Groot M, Samson JA. The evaluation of two measures of quality of life in patients with type I and type II diabetes. Diabetes Care. 1994; 17:267-274. [PubMed: 8026281]

29. Beck, AT.; Steer, R.; Brown, G. Beck Depression Inventory. 2. San Antonio, Tex: The Psychological Corporation; 1996.

30. Taub LF, Redeker NS. Sleep disorders, glucose regulation, and type 2 diabetes. Biol Res Nurs. 2008; 9:231-243. [PubMed: 18077776]

31. Einhorn D, Stewart DA, Erman MK, Gordon N, Philis-Tsimikas A, Casal E. Prevalence of sleep apnea in a population of adults with type 2 diabetes mellitus. Endocr Pract. 2007; 13:355-362. [PubMed: 17669711]

32. Merlino G, Valente M, Serafini A, et al. Effects of restless legs syndrome on quality of life and psychological status in patients with type 2 diabetes. Diabetes Educ. 2010; 36:79-87. [PubMed: 20185611]

33. Cuellar NG, Ratcliffe SJ. A comparison of glycemic control, sleep, fatigue, and depression in type 2 diabetes with and without restless legs syndrome. J Clin Sleep Med. 2008; 4:50-56. [PubMed: 18350963]

34. Strine TW, Chapman DP. Associations of frequent sleep insufficiency with health-related quality of life and health behaviors. Sleep Med. 2005; 6:23-27. [PubMed: 15680291]

35. Chasens ER. Understanding sleep in persons with diabetes. Diabetes Educ. 2007; 33:435-441. [PubMed: 17570874] 
Table 2

Correlations Between PSQI and the Other Continuous Variables

\begin{tabular}{|lc|}
\hline Variable & Global PSQI \\
\hline Age & -0.10 \\
Years since diabetes diagnosis & -0.02 \\
No. of comorbidities & $0.29^{a}$ \\
No. of diabetic complications & 0.11 \\
BDI-II & $0.48^{a}$ \\
SF-36 MCS & $-0.35^{a}$ \\
SF-36 PCS & $-0.33^{a}$ \\
Physical functioning & $-0.30^{a}$ \\
Role, physical & $-0.31^{a}$ \\
Bodily pain & $-0.39 a$ \\
General health & $-0.40^{a}$ \\
Vitality & $-0.47^{a}$ \\
Social functioning & $-0.34^{a}$ \\
Role, emotional & $-0.33^{a}$ \\
Mental health & $-0.42^{a}$ \\
DQOL total & $0.39^{a}$ \\
DQOL satisfaction & $0.44^{a}$ \\
DQOL impact & $0.42^{a}$ \\
DQOL diabetes-related worry & $0.28^{a}$ \\
\hline
\end{tabular}

BDI-II, Beck Depression Inventory-II; DQOL, Diabetes Quality of Life measure; MCS, SF-36 Mental Component Summary score; PCS, SF-36 Physical.

Component Summary score.

${ }^{a} P<.001$. 


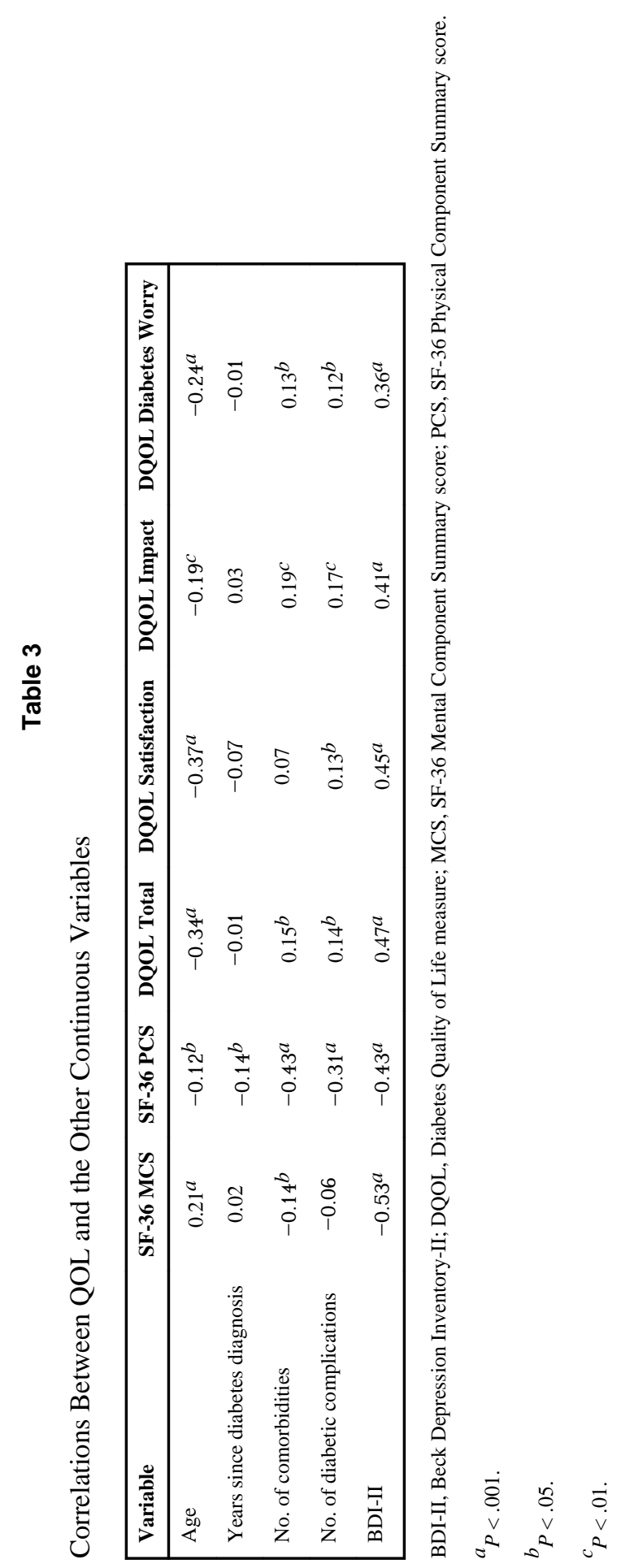

Diabetes Educ. Author manuscript; available in PMC 2011 November 18. 


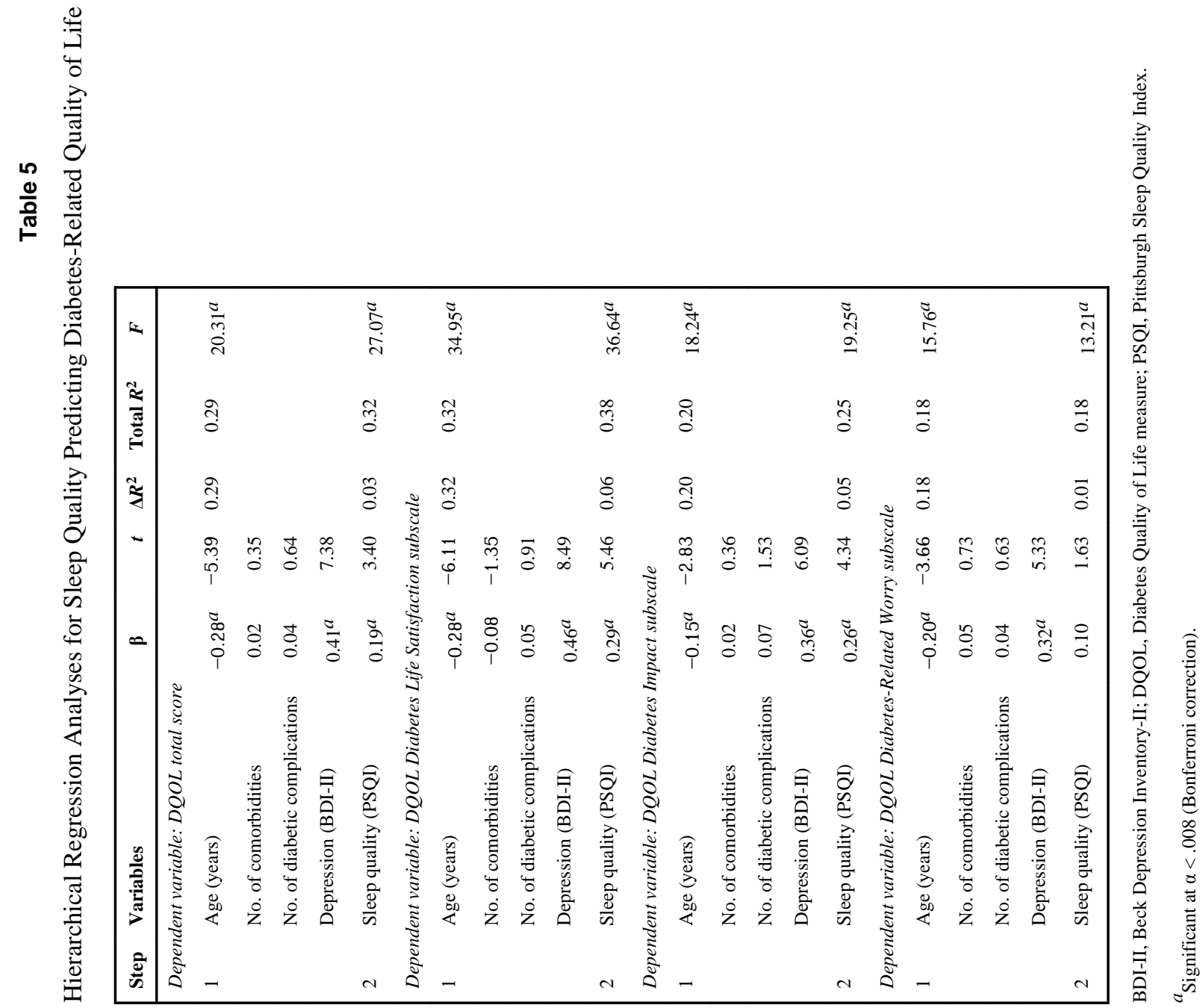

Diabetes Educ. Author manuscript; available in PMC 2011 November 18. 